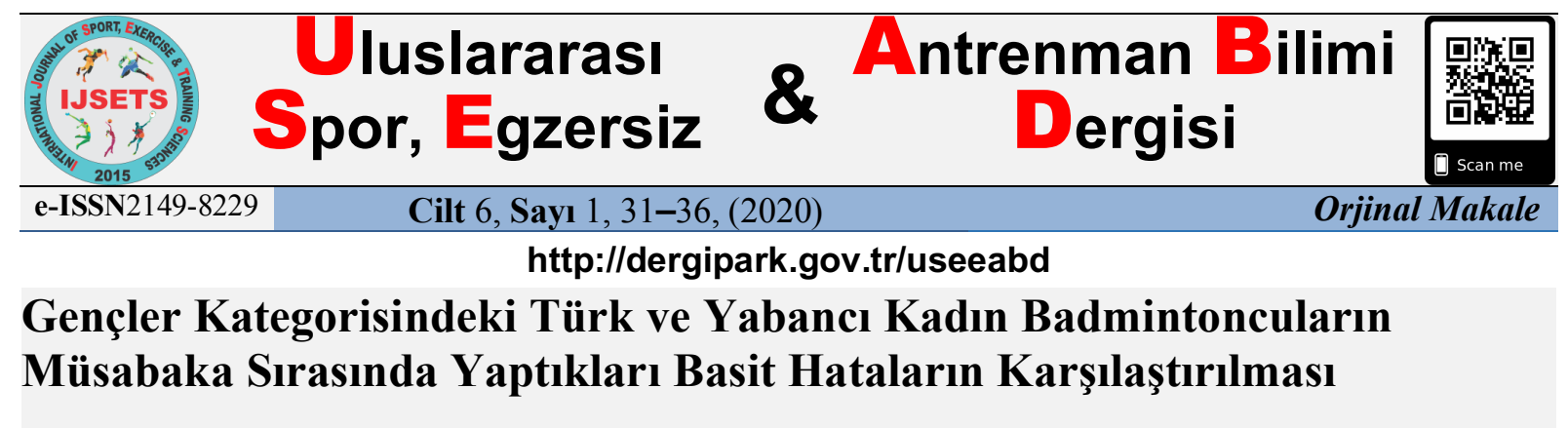

Beyhan ÖZGÜR ${ }^{1}$ Özet
Amaç: Bu çalışmada, gençler kategorisindeki Türk ve yabancı kadın badmintoncuların
müsabaka sırasında yaptıkları basit hataların karşılaştırılması amaçlanmıştır.
Materyal ve Metot: Araştırmaya, 22 Türk (yaş $16,04 \pm 0,88$ yıl) ve 10 yabancı (yaş
$16,24 \pm 0,66$ yıl) badminton oyuncusu katılmıştır. Çalışmada 45 tek kadın maçı ( 28 Türk, 17
yabancı) analiz edilmiştir. Basit hatalar kort bölgelerine göre (ön, orta ve arka kort) aut ve file
hatası olarak analiz edilmiştir. Katılımcıların tanımlayıcı istatistikleri için ortalama ve standart
sapma değerleri hesaplanmıştır. Türk ve yabancı oyuncular arasındaki farkı tespit etmek için
Mann-Whitney U ve Bağımsız grup t-test kullanılmıştır. Ayrıca, Türk ve yabancı oyuncuların
galip ve mağlup olma durumlarına göre aralarındaki farkı incelemek için Kruskal-Wallis H
testi kullanılmıştır.
Bulgular: Araştırma sonuçlarına göre Türk ve yabancı badmintoncuların 1. set, 2. set ve maç
genelindeki tüm basit hata değerleri benzer bulunmuştur (p>0,05). Mağlup yabancı
sporcuların basit hata değerleri galip Türk ve yabancı sporcuların basit hata değerlerinden
anlamlı olarak daha az bulunmuştur (p<0,05).
Sonuç: Türk ve yabancı kadın badmintoncuların müsabaka sırasındaki yaptıkları basit hata
değerleri benzer görülürken, mağlup sporcuların galip sporculardan daha düşük basit hata
oranı gösterdileri bulunmuştur. Bu nedenle, basit hataların müsabaka performansına etkisinin
tam olarak anlaşılabilmesi için gelecek çalışmalarda zorunlu hatalar ve direk sayılara yönelik
analizlerin de yapılması önerilmektedir. Anahtar Kelimeler

Badminton,

Basit hata,

Müsabaka,

Karşılaştırma,

Yayın Bilgisi

Gönderi Tarihi: 26.12 .2019

Kabul Tarihi: 10.03 .2020

Online Yayın Tarihi: 20.03.2020

DOI:10.18826/useeabd.665657

\title{
Comparison of Unforced Errors of Turkish and Foreign Female Badminton Players during the Competition in the Youth Category
}

$\begin{array}{lr}\text { Abstract } & \text { Keywords } \\ \text { Aim: In this study, it has been aimed to compare the unforced errors made by Turkish and } & \text { Badminton, } \\ \text { foreign female badminton players during the competition in the youth category. } & \text { Unforced error, } \\ \text { Methods: } 22 \text { Turkish (age } 16.04 \pm 0.88 \text { ) and } 10 \text { foreign (age } 16.24 \pm 0.66) \text { badminton players } & \text { Competition, } \\ \text { have participated in the study. In this study, } 45 \text { women singles match (28 Turkish, } 17 \text { foreign) } & \text { Comparison, }\end{array}$

have been analyzed. Unforced errors have been analyzed as out and net errors according to the court areas (front, middle and back). Mean and standard deviation values have been calculated for the descriptive statistics of the participants. Mann-Whitney U and IndependentSamples T-tests have used to determine the difference between Turkish and foreign players. In addition, the Kruskal-Wallis $\mathrm{H}$ test has been used to examine the difference between Turkish and foreign athletes who won and lost the match.

Results: According to the results of the study, all unforced error values of Turkish and foreign badminton players in the first set, second set and match have been found to be similar $(p>0.05)$. Losing foreign athletes have made significantly less unforced errors than winning Turkish and foreign athletes $(\mathrm{p}<0.05)$.

Conclusion: While Turkish and foreign female badminton players had similar simple error values during the competition, losers showed lower simple error rates than the winners. Therefore, in order to fully understand the effect of unforced errors on the performance of the competition, it is recommended that future studies should also carry out analyzes for forced errors and direct points.

\section{GíRiș}

Badminton, karşılıklı iki ve ya dört kişi ile kapalı salonda oynanan, özellikle Olimpiyat Oyunları'na (1992 Barselona) dahil edilmesinden bu yana popülerlik kazanmış bir raket sporudur (Lin, 2014). Oyuncular, ortasından file ile bölünmüş $80 \mathrm{~m}^{21}$ lik bir kortta raket ile kaz tüyünden yapılmış topa farklı teknikler ile vuruş yaparak sayı kazanmaya çalışır (Faude vd., 2007; Paterson, McMaster \& Cronin, 2016; Sonoda vd., 2018). Bu teknikler, kortta kullanıldıkları bölgelerde göre faklılık göstermektedir. Örneğin, net drop, lob ve net kill ön kort, drive ve savunma orta kort, clear, drop ve smaç arka kort

${ }^{1}$ High School of Physical Education and Sports, Mardin Artuklu University, Mardin/Turkey, ORCID ID: 0000-0002-7813-4119 
bölgelerinde uygulanan tekniklerdir (Larsen, 2006). Ayrıca badminton, hızlı ivmelenmeler, yavaşlamalar ve kısa mesafelerdeki hızlı yön değişimlerinin de dahil olduğu birçok yüksek yoğunluklu hareket içeren bir spordur (Sturgess \& Newton, 2008; Nadzalan, Mohamad \& Chinnasee, 2018). Karşılaşmalarda sporcuların bir sayı kazanabilmeleri için ralliler esnasında $400 \mathrm{~km} / \mathrm{s}$ 'yi aşan tüy topun hızına, yoğun ve şiddetli tempolara ayak uydurmaları gerekmektedir. Olimpiyat Oyunları'nda yapılan

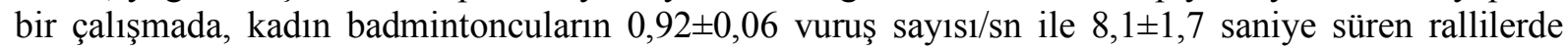
$7,4 \pm 1,7$ vuruş yaptıkları ifade edilmiştir (Abian-Vicen, Castanedo, Abian \& Sampedro, 2013). Ayrıca oyuncuların müsabakalarda 68-83 ralli ve 500-800 vuruş gerçekleştirdikleri de bildirilmiştir (Chiminazzo, Barreira, Luz, Saraiva \& Cayres, 2018; Cabello, Padial, Lees \& Rivas, 2004; Abdullahi \& Coetzee, 2017). Branşın bu yüksek hız ve şiddetinden dolayı badmintoncuların, çeviklik, dayanıklılık, esneklik, güç ve hızlanma gibi fiziksel performans yetkinlikleri bakımından üstün olmaları gereklidir (Paterson vd., 2016).

Badmintonda ralliler genellikle; direk sayılar, zorunlu hatalar ve basit hatalar şeklinde sonuçlanır. Son yıllarda yapılan araştırmalar, özellikle basit hataların (oyuncuların herhangi bir zor pozisyonda olmadığı halde yaptığı hatalar) yüksek bir yüzdeye sahip olduğuna işaret etmektedir (Abian, Castanedo, Feng, Sampedro \& Abian-Vicen, 2014; Laffaye, Phomsoupha \& Dor, 2015). Örneğin, Laffaye ve diğerleri (2015) 1992-2012 yılları arasında düzenlenen olimpiyat oyunlarının final müsabakalarında yapılan basit hata oranını \% 42 olarak raporlamışlardır. Diğer bir çalışmada ise ulusal düzeyde Tayvanlı oyuncuların basit hata oranı \% 65 olarak bulunmuştur (Chen \& Chen, 2008). Bazı araştırmacılar ise kazanan ve kaybeden oyuncuların basit hata değerlerini incelemiş ve kazanan oyuncuların daha az basit hata yaptığını bildirmişlerdir (Yadav \& Shukla, 2011; Cabello-Manrique \& González-Badillo, 2003). Bununla birlikte, literatürde Türk sporcular ile yabanc1 sporcuların müsabaka ortamında yaptıkları basit hatalar ile ilgili araştırmalar oldukça sınırlıdır. Daha önceki bir araştırmada, Türk ve yabancı erkek sporcuların basit hata değerleri karşılaştırılmıştır (Özgür, 2019a). Ancak, kadın sporcuların yabancı sporcularla kıyaslandığı her hangi bir çalışma ele alınmamıştır. Bu nedenle çalışmada, Türk ve yabancı kadın badmintoncuların müsabaka ortamında yaptıkları basit hataların karşılaştırılması amaçlanmıştır.

\section{MATERYAL ve YÖNTEM}

\section{Katılımcilar}

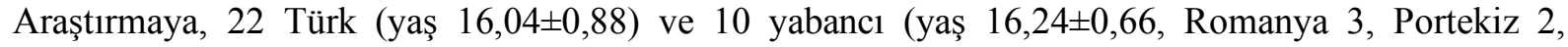
Ukrayna 2, Hindistan 1, Rusya 1, Bulgaristan 1) badminton oyuncusu katılmıştır. Çalışma, 13-16 Aralık 2017' de Ankara' da düzenlenen Uluslararası gençler badminton turnuvasında (Turkey Junior 2017) gerçekleştirilmiştir.

\section{Basit Hata Analizi}

Basit hata analizi için toplamda 45 tek kadın maçı (28 Türk, 17 yabancı) analiz edilmiştir. Basit hata analizi; KTS (kaybedilen toplam sayı), BKTSY (basit hataların kaybedilen toplam sayıdaki yüzdesi), TH (toplamda yapılan basit hata), TAH (toplam aut hatas1), TFH (toplam file hatas1), HÖK (hata ön kort), HOK (hata orta kort), HAK (hata arka kort), HAÖK (hata aut ön kort), HAOK (hata aut orta kort), HAAK (hata aut arka kort), HFÖK (hata file ön kort), HFOK (hata file orta kort), HFAK (hata file arka kort) olarak yapılmıştır. Müsabaka sırasında araştırma grubunun maçları izlenerek, basit hatalar, yapıldığı bölgeye göre basit hata analiz tablosuna işlenmiştir. Analizler 1. set, 2. Set, maç geneli, galip ve mağlup olma durumuna göre yapılmıştır.

\section{İstatiksel Analiz}

Veriler, SPSS 22 paket programı kullanılarak analiz edilmiştir. Katılımcıların tanımlayıcı istatistikleri için ortalama ve standart sapma değerleri hesaplanmıştır. Shapiro Wilk-W testi kullanılarak verilerin normal dağılıma sahip olup olmadığı incelenmiştir. Test sonucuna göre bazı değişkenlerin normal dağılıma sahip olmadığı, bazı değişkenlerin ise normal dağılım gösterdiği tespit edilmiştir. Türk ve yabancı oyuncular arasındaki farkı tespit etmek için normal dağılım göstermeyen veriler Mann Whitney-U, normal dağılım gösteren veriler ise Bağımsız grup t-test kullanılarak analiz edilmiştir. Ayrıca, Türk ve yabancı oyuncuların galip ve mağlup olma durumuna göre aralarındaki farkı incelemek için Kruskal-Wallis testi kullanılmıştır. 


\section{BULGULAR}

Tablo 1. Türk ve yabancı badmintoncuların 1. sette yaptıkları basit hata değerleri

\begin{tabular}{lccc}
\multicolumn{1}{c}{ Değişkenler } & $\begin{array}{c}\text { Türk } \\
\text { Maç }(\mathbf{n = 2 8}) \overline{\boldsymbol{X}} \pm \mathbf{S S}\end{array}$ & $\begin{array}{c}\text { Yabanci } \\
\mathbf{M a c ̧}(\mathbf{n}=\mathbf{1 7}) \overline{\boldsymbol{X}} \pm \mathbf{S S}\end{array}$ & $\mathbf{p}$ \\
\hline KTS & $17,82 \pm 4,11$ & $16,41 \pm 6,00$ & $0,333^{\mathbf{M}}$ \\
BKTSY & $46,47 \pm 20,16$ & $46,51 \pm 23,45$ & $0,996^{\mathbf{B}}$ \\
TH & $8,21 \pm 3,79$ & $7,47 \pm 4,71$ & $0,564^{\mathbf{B}}$ \\
TAH & $4,93 \pm 2,72$ & $4,35 \pm 2,93$ & $0,508^{\mathbf{B}}$ \\
TFH & $3,18 \pm 2,29$ & $3,12 \pm 2,71$ & $0,758^{\mathbf{M}}$ \\
HÖK & $3,53 \pm 2,44$ & $2,82 \pm 2,15$ & $0,328^{\mathbf{B}}$ \\
HOK & $1,46 \pm 1,62$ & $1,76 \pm 1,64$ & $0,507^{\mathbf{M}}$ \\
HAK & $3,10 \pm 1,98$ & $2,88 \pm 2,08$ & $0,720^{\mathbf{B}}$ \\
HAÖK & $2,18 \pm 2,09$ & $1,82 \pm 1,46$ & $0,543^{\mathbf{B}}$ \\
HAOK & $0,79 \pm 1,19$ & $0,53 \pm 0,71$ & $0,693^{\mathbf{M}}$ \\
HAAK & $2,11 \pm 1,44$ & $2,00 \pm 1,80$ & $0,616^{\mathbf{M}}$ \\
HFÖK & $1,36 \pm 1,39$ & $1,00 \pm 1,36$ & $0,271^{\mathbf{M}}$ \\
HFOK & $0,68 \pm 0,94$ & $1,24 \pm 1,25$ & $0,110^{\mathbf{M}}$ \\
HFAK & $1,00 \pm 1,33$ & $0,88 \pm 1,05$ & $0,910^{\mathbf{M}}$ \\
\hline
\end{tabular}

B: Bağımsız grup t-test; M: Mann Whitney-U

Tablo 2. Türk ve yabancı badmintoncuların 2. sette yaptıkları basit hata değerleri

\begin{tabular}{lccc}
\multicolumn{1}{c}{ Değişkenler } & $\begin{array}{c}\text { Türk } \\
\text { Maç }(\mathbf{n}=\mathbf{2 8}) \overline{\boldsymbol{X}} \pm \mathbf{S S}\end{array}$ & $\begin{array}{c}\text { Yabancı } \\
\text { Mac }(\mathbf{n}=\mathbf{1 7}) \overline{\boldsymbol{X}} \pm \mathbf{S S}\end{array}$ & $\mathbf{p}$ \\
\hline KTS & $17,25 \pm 5,11$ & $15,47 \pm 5,62$ & $0,267^{\mathbf{M}}$ \\
BKTSY & $47,85 \pm 21,42$ & $42,82 \pm 15,47$ & $0,404^{\mathbf{B}}$ \\
TH & $8,21 \pm 3,97$ & $6,18 \pm 2,62$ & $0,068^{\mathbf{B}}$ \\
TAH & $4,46 \pm 3,01$ & $3,06 \pm 2,13$ & $0,131^{\mathbf{M}}$ \\
TFH & $3,75 \pm 2,47$ & $3,12 \pm 2,08$ & $0,384^{\mathbf{B}}$ \\
HÖK & $3,42 \pm 2,57$ & $2,70 \pm 2,25$ & $0,292^{\mathbf{M}}$ \\
HOK & $1,71 \pm 1,69$ & $1,58 \pm 1,66$ & $0,810^{\mathbf{M}}$ \\
HAK & $3,00 \pm 2,10$ & $2,05 \pm 1,95$ & $0,143^{\mathbf{B}}$ \\
HAÖK & $2,11 \pm 2,23$ & $1,18 \pm 1,23$ & $0,210^{\mathbf{M}}$ \\
HAOK & $0,61 \pm 1,06$ & $0,65 \pm 0,93$ & $0,650^{\mathbf{M}}$ \\
HAAK & $1,68 \pm 1,46$ & $1,29 \pm 1,72$ & $0,250^{\mathbf{M}}$ \\
HFÖK & $1,32 \pm 1,24$ & $1,53 \pm 1,87$ & $0,874^{\mathbf{M}}$ \\
HFOK & $1,11 \pm 1,22$ & $0,94 \pm 1,19$ & $0,653^{\mathbf{M}}$ \\
HFAK & $1,32 \pm 1,51$ & $0,76 \pm 1,03$ & $0,193^{\mathbf{M}}$ \\
\hline
\end{tabular}

B: Bağımsız grup t-test; M: Mann Whitney-U

Türk ve yabancı oyuncuların 1 ve 2 sette yaptıkları basit hata değerleri benzer bulunmuştur $(\mathrm{p}>0.05)$.

Tablo 3. Türk ve yabancı badmintoncuların maç genelinde yaptıkları basit hata değerleri

\begin{tabular}{lccc}
\hline Değişkenler & $\begin{array}{c}\text { Türk } \\
\text { Maç (n=28) } \overline{\boldsymbol{X}} \pm \text { SS }\end{array}$ & $\begin{array}{c}\text { Yabanci } \\
\text { Maç (n=17) } \mathbf{X} \pm \text { SS }\end{array}$ & $\mathbf{p}$ \\
\hline KTS & $35,07 \pm 8,71$ & $31,88 \pm 10,42$ & $0,275^{\mathbf{B}}$ \\
BKTSY & $46,96 \pm 16,50$ & $45,29 \pm 17,44$ & $0,749^{\mathbf{B}}$ \\
TH & $16,43 \pm 6,64$ & $13,65 \pm 5,65$ & $0,158^{\mathbf{B}}$ \\
TAH & $9,39 \pm 4,77$ & $7,41 \pm 3,06$ & $0,134^{\mathbf{B}}$ \\
TFH & $6,93 \pm 4,07$ & $6,24 \pm 4,36$ & $0,593^{\mathbf{B}}$ \\
HÖK & $6,96 \pm 4,21$ & $5,52 \pm 3,64$ & $0,251^{\mathbf{B}}$ \\
HOK & $3,17 \pm 2,73$ & $3,35 \pm 2,69$ & $0,836^{\mathbf{B}}$ \\
HAK & $6,10 \pm 3,07$ & $4,94 \pm 3,15$ & $0,228^{\mathbf{M}}$ \\
HAÖK & $4,29 \pm 3,72$ & $3,00 \pm 2,00$ & $0,197^{\mathbf{B}}$ \\
HAOK & $1,39 \pm 1,64$ & $1,18 \pm 1,46$ & $0,704^{\mathbf{M}}$ \\
HAAK & $3,79 \pm 1,66$ & $3,29 \pm 2,54$ & $0,437^{\mathbf{B}}$ \\
HFÖK & $2,68 \pm 1,98$ & $2,53 \pm 2,78$ & $0,272^{\mathbf{M}}$ \\
HFOK & $1,79 \pm 1,89$ & $2,18 \pm 2,00$ & $0,492^{\mathbf{M}}$ \\
HFAK & $2,32 \pm 2,21$ & $1,65 \pm 1,69$ & $0,302^{\mathbf{M}}$ \\
\hline
\end{tabular}

B: Bağımsız grup t-test; M: Mann Whitney-U 
Türk ve yabancı oyuncuların maç genelinde yaptıkları basit hata değerleri benzer bulunmuştur $(\mathrm{p}>0.05)$.

Tablo 4. Türk ve yabancı badmintoncuların galip ve mağlup olma durumuna göre basit hata değerleri

\begin{tabular}{|c|c|c|c|c|c|}
\hline \multirow[b]{2}{*}{ Değişkenler } & \multicolumn{2}{|c|}{ Galip Galip } & \multicolumn{2}{|c|}{ Mağlup Mağlup } & \multirow[b]{2}{*}{$\mathbf{p}$} \\
\hline & $\begin{array}{c}\text { Türk } \\
\operatorname{Mac}(\mathrm{n}=12) \bar{X} \pm \mathrm{SS}\end{array}$ & $\begin{array}{c}\text { Yabanci } \\
\operatorname{Mac}(\mathbf{n}=12) \bar{X} \pm \mathrm{SS}\end{array}$ & $\begin{array}{c}\text { Türk } \\
\operatorname{Mac}(\mathrm{n}=16) \bar{X} \pm S S\end{array}$ & $\begin{array}{c}\text { Yabancı } \\
\operatorname{Mac}(n=5) \bar{X} \pm S S\end{array}$ & \\
\hline KTS & $26,83 \pm 7,05^{\mathrm{CD}}$ & $28,33 \pm 10,14^{\mathrm{CD}}$ & $41,25 \pm 2,26^{\mathbf{A B}}$ & $40,40 \pm 4,77^{\mathbf{A B}}$ & $0,000 * *$ \\
\hline BKTSY & $49,51 \pm 21,68^{\mathbf{D}}$ & $52,90 \pm 14,47^{\mathbf{D}}$ & $45,04 \pm 11,66$ & $27,02 \pm 7,16^{\mathbf{A B}}$ & $0,015 *$ \\
\hline $\mathrm{TH}$ & $13,42 \pm 7,31$ & $14,83 \pm 6,20$ & $18,69 \pm 5,23$ & $10,80 \pm 2,77$ & $0,025 *$ \\
\hline $\mathrm{TAH}$ & $8,33 \pm 5,58$ & $7,75 \pm 3,46$ & $10,19 \pm 4,07$ & $6,60 \pm 1,81$ & 0,167 \\
\hline $\mathrm{TFH}$ & $4,83 \pm 2,36$ & $7,08 \pm 4,71$ & $8,50 \pm 4,42$ & $4,20 \pm 2,77$ & 0,071 \\
\hline HÖK & $6,00 \pm 4,22$ & $6,16 \pm 4,04$ & $7,68 \pm 4,19$ & $4,00 \pm 2,00$ & 0,342 \\
\hline HOK & $2,08 \pm 2,15$ & $3,91 \pm 2,81$ & $4,00 \pm 2,89$ & $2,00 \pm 2,00$ & 0,149 \\
\hline HAK & $5,00 \pm 2,59$ & $4,91 \pm 2,90$ & $6,93 \pm 3,21$ & $5,00 \pm 4,06$ & 0,326 \\
\hline HAÖK & $3,83 \pm 3,68$ & $3,25 \pm 2,13$ & $4,63 \pm 3,82$ & $2,40 \pm 1,67$ & 0,602 \\
\hline HAOK & $1,08 \pm 1,56$ & $1,50 \pm 1,62$ & $1,62 \pm 1,70$ & $0,40 \pm 0,54$ & 0,371 \\
\hline HAAK & $3,50 \pm 1,67$ & $3,08 \pm 2,39$ & $4,00 \pm 1,67$ & $3,80 \pm 3,11$ & 0,597 \\
\hline HFÖK & $2,17 \pm 1,69$ & $2,92 \pm 3,17$ & $3,06 \pm 2,14$ & $1,60 \pm 1,34$ & 0,381 \\
\hline HFOK & $1,00 \pm 1,04$ & $2,42 \pm 2,19$ & $2,38 \pm 2,18$ & $1,60 \pm 1,51$ & 0,299 \\
\hline HFAK & $1,50 \pm 1,24$ & $1,83 \pm 1,85$ & $2,94 \pm 2,59$ & $1,20 \pm 1,30$ & 0,331 \\
\hline
\end{tabular}

Türk ve yabancı oyuncuların galip ve mağlup olma durumuna göre KTS, BKTSY ve TH değerleri arasında anlamlı fark bulunmuştur $(\mathrm{p}<0.05)$.

\section{TARTIŞMA}

$\mathrm{Bu}$ çalışmada Türk ve yabancı kadın badmintoncuların müsabaka ortamında yaptıkları basit hata değerleri incelenmiştir. Araştırma sonuçlarına göre Türk ve yabancı badmintoncuların 1. set, 2. set ve maç genelindeki tüm basit hata değerleri benzer bulunmuştur $(\mathrm{p}>0,05)$. Basit hataların, dikkat, konsantrasyon, teknik, taktiksel ve fiziksel performans seviyesindeki yetersizlikten kaynaklanabileceği ifade edilmektedir (Yadav \& Shukla, 2011; Omveer, 2017; Hotaman, Özgür \& Coşan, 2018). Bu çalışmanın sonuçları, Türk ve yabancı sporcuların ifade edilen bu özellikler bakımından aynı seviyede olduklarını göstermektedir. Literatüredeki çalışmalara bakıldığında; Abian-Vicen ve diğerleri (2013) Pekin Olimpiyat Oyunları'nda yaptıkları analiz çalışmasında kadın sporcuların yaptığı basit hata oranını \% 48,6 $\pm 9,0$ olarak rapor etmişlerdir. Bu araştırmadaki Türk $(\% 46,96 \pm 16,50)$ ve yabancı $(45,29 \pm 17,44)$ badmintoncuların basit hata değerleri Olimpiyat Oyunları'na katılma hakk1 elde eden üst seviyedeki sporculardan daha düşük bulunmuştur. Olimpiyat Oyunları'na katılma hakkı kazanan sporcuların tekniksel, taktiksel ve psikolojik olarak daha iyi seviyede oldukları düşünüldüğünden daha az basit hata yapmaları beklenmektedir. Ancak burada turnuva seviyeleri de göz önünde bulundurulmalıdır. Olimpiyat Oyunları'na kota alarak katılma hakkı elde eden sınırlı sayıda sporcunun katıldığı göz önüne alındığında, sporcuların üzerindeki baskının diğer turnuvalara göre çok daha fazla olduğu düşünülmektedir. Bu nedenle çalışmamızdaki verilerin literatürden farklı olması turnuva seviyelerindeki farklılığa bağlanabilir.

Çalışmanın diğer konusu da galip ve mağlup sporcuların basit hata değerlerinin incelenmesidir. Çalışmanın sonuçlarına göre mağlup yabancı sporcuların basit hata değerleri galip Türk ve yabancı sporcuların basit hata değerlerinden anlamlı olarak daha az bulunmuştur $(\mathrm{p}<0,05)$. Literatürdeki daha önceki araştırmalara bakıldığında; Tong ve Hong (2000) kazanan oyuncuların (\% 21,20土10,27) kaybeden oyuculardan $(\% 25,30 \pm 8,23)$ daha az basit hata yaptıklarını bulmuştur. Özgür $(2019 b) 17$ yaş grubunda toplam hata, orta kort bölgesinde ve toplamda daha az file hatas1 yapan kadın sporcuların müsabakaları galip olarak bitirdiklerini bildirilmiştir. Diğer bir çalışmada kazanan sporcuların \% 73' ünün müsabakaları daha düşük basit hata yüzdesi ile bitirdiği rapor edilmiştir (Cabello-Manrique \& González-Badillo, 2003). Benzer şekilde, U19 grubunda orta kort bölgesinde ve toplamda daha az aut hatası yapan erkek sporcuların müsabakaları galip olarak bitirdikleri bildirilmiştir (Özgür, 2018). Çalışmadaki bu sonuçlar literatür ile farkl1lık göstermektedir. Bu farklılık, çalışmadaki yabancı mağlup sporcuların kaybettikleri sayıların çoğunluğunun zorunlu hatalar ve direk 
sayılardan meydana geldiğini göstermektedir. Sporcuların direk sayılardan dolayı kaybettikleri sayılar savunmadaki, zorunlu hatalardan dolayı kaybettikleri sayılar ise adımlama hareketlerindeki yetersizliğe işaret etmektedir.

\section{SONUÇ ve ÖNERILER}

Gençler kategorisindeki Türk ve yabancı kadın badmintoncuların müsabaka sırasındaki yaptıkları basit hata değerleri benzer bulunurken, Mağlup yabancı sporcular, galip Türk ve yabancı sporculardan anlamlı şekilde daha düşük basit hata oranı göstermişlerdir. Basit hataların müsabaka performansına etkisinin tam olarak anlaşılabilmesi için gelecek çalışmalarda zorunlu hatalar ve direk sayılar ile kaybedilen sayılara yönelik analizler de yapılmalıdır.

\section{PRATIK/SAHA UYGULAMALARI}

Badminton atrenörleri müsabakalarda düzenli olarak basit hata analizi yaparak değerlendirmeler yapabilir. Bu değerlendirmelere göre basit hatanın yapıldığı bölge (ön, orta, arka kort) ve yapılış şekli (aut ve file hatası) tespit edilebilir. Bu tespitlere göre de özel antrenman programları oluşturulup sporcuların yaptıkları basit hataların azaltılmasına katkı sağlanabilir.

\section{KAYNAKÇA}

Abdullahi, Y. \& Coetzee, B. (2017). Notational singles match analysis of male badminton players who participated in the African Badminton Championships. International Journal of Performance Analysis in Sport, 17(1-2), 1-16.

Abian, P., Castanedo, A., Feng, X. Q., Sampedro, J. \& Abian-Vicen, J. (2014) Notational comparison of men's singles badminton matches between Olympic Games in Beijing and London. International Journal of Performance Analysis in Sport, 14(1), 42-53

Abian-Vicen, J., Castanedo, A., Abian, P. \& Sampedro, J. (2013). Temporal and notational comparison of badminton matches between men's singles and women's singles. International Journal of Performance Analysis in Sport, 13(2), 310-320.

Chen, H. L. \& Chen, T. C. (2008). Temporal structure comparison of the new and conventional scoring systems for men's badminton singles in Taiwan. Journal of Exercise Science and Fitness, $6(1), 34-43$.

Chiminazzo, J. G. C., Barreira, J., Luz, L. S. M., Saraiva, W. C. \& Cayres, J. T. (2018). Technical and timing characteristics of badminton men's single: comparison between groups and play-offs stages in 2016 Rio Olympic Games. International Journal of Performance Analysis in Sport, 18(2), 245-254.

Cabello, D., Padial, P., Lees, A. \& Rivas, F. (2004). Temporal and physiological characteristics of elite women and men singles badminton. International Journal of Applied Sports Sciences, 16, 112.

Cabello-Manrique, D. \& Gonzalez-Badillo, J. J. (2003). Analysis of the characteristics of competitive badminton. British Journal of Sports Medicine, 37(1), 62-66.

Faude, O., Meyer, T., Rosenberger, F., Fries, M., Huber, G. \& Kindermann, W. (2007). Physiological characteristics of badminton match play. Eur J Appl Physiol, 100, 479-485.

Hotaman, F., Özgür, B. \& Coşan, F. (2018). 17 yaş grubu milli badmintoncuların müsabaka sırasında yaptıkları basit hatalar ile fiziksel performansları arasındaki ilişki. Spor Bilimleri Araştırmaları Dergisi, 3(1), 73-84.

Laffaye, G., Phomsoupha, M. \& Dor, F. (2015). Changes in the game characteristics of a badminton match: a longitudinal study through the Olympic game finals analysis in men's singles. $J$ Sports Sci Med, 14(3), 584-590.

Larsen, K. (2006). Badminton Europe coach education level 3. Denmark: Broendby.

Lin, R.Z. (2014). Neuromuscular fatigue following a singles badminton match (Yüksek lisans tezi). Erişim adresi: http://ro.ecu.edu.au/theses/1279 
Nadzalan, A. M., Mohamad, N. I., Lee, J. L. F. \& Chinnasee, C. (2018). Relationship between muscle architecture and badminton-specific physical abilities. Human Movement, 19(1), 44-50.

Omveer. (2017). A study on prediction of playing ability in badminton from selected anthropometrical physical and physiological characteristics among inter collegiate players. International Journal of Advanced Research and Development, 2(5), 50-54.

Özgür, B. (2018). U17 ve U19 erkek milli badmintoncuların müsabaka ortamında yaptıkları basit hataların karşılaştırılması. Ulusal spor bilimleri dergisi, 2(2), 138-145.

Özgür, B. (2019a). Elit genç Türk ve yabanci milli badminton oyuncularının müsabaka sırasında yaptıkları basit hataların analizi. SPORMETRE Beden Eğitimi ve Spor Bilimleri Dergisi, 17(3), $145-152$.

Özgür, B. (2019b). 17 ve 19 yaş altı kadın milli badminton oyuncularının müsabaka sırasında yaptıkları basit hataların karşılaştııılması. Spor ve Performans Araştırmaları Dergisi, 10(2), 123 130.

Paterson, S., McMaster, D. T. \& Cronin, J. (2016). Assessing change of direction ability in badminton athletes. Strength and Conditioning Journal, 38(5), 18-30.

Sonoda, T., Tashiro, Y., Suzuki, Y., Kajiwara, Y., Zeidan, H., Yokota, Y., ... Aoyama, T. (2018). Relationship between agility and lower limb muscle strength, targeting university badminton players. $J$ Phys Ther Sci, 30, 320-323.

Sturgess, S. \& Newton, R. U. (2008). Design and implementation of a specific strength program for badminton. Strength Cond J, 30(3), 33-41.

Tong, Y. M. \& Hong, Y. (2000). The playing pattern of world's top single badminton players. 18th International Symposium on Biomechanics in Sports, (s. 1-6). Hong Kong.

Yadav, S. K. \& Shukla, Y. M. (2011). Analysis of unforced errors in relation to performance in singles in badminton. International Journal of Physical Education, 4(2), 117-119.

\section{CITATION OF THIS ARTICLE}

Özgür, B. (2019). Comparison of Unforced Errors of Turkish and Foreign Female Badminton Players during the Competition in the Youth Category. International Journal of Sport, Exercise \& Training Sciences - IJSETS, 6(1), 31-36. DOI: 10.18826/useeabd.665657 